\title{
Multi Pulse Pump-Probe Diagnostics for Development of Advanced Transparent Materials Processing
}

\author{
M. Jenne, ${ }^{1,2}$ F. Zimmermann, ${ }^{1}$ D. Flamm, ${ }^{1}$ D. Großmann, ${ }^{1,3}$ J. Kleiner, ${ }^{1}$ M. Kumkar, ${ }^{1}$ S. Nolte ${ }^{2,4}$ \\ ${ }^{1}$ TRUMPF Laser- und Systemtechnik GmbH, Johann-Maus-Straße 2, 71254 Ditzingen, Germany \\ E-mail: michael.jenne@trumpf.com \\ ${ }^{2}$ Institute of Applied Physics, Friedrich-Schiller-University Jena, Max-Wien-Platz 1, 07743 Jena, \\ Germany \\ ${ }^{3}$ Chair for Laser Technology, Technical University Aachen, Steinbachstr. 15, 52074 Aachen, \\ Germany \\ ${ }^{4}$ Fraunhofer Institute for Applied Optics and Precision Engineering, Albert-Einstein-Straße 7, \\ 07745 Jena, Germany
}

\begin{abstract}
Time-resolved pump-probe microscopy experiments are presented to demonstrate the impact of laser-processing parameters onto the fundamental interaction processes between ultrashort laser pulses and glass. Our approach includes the implementation of polarization microscopy to detect the transient buildup of pressure waves and stress from single-shot and multi-pulse experiments. The presented data gives insight into the interplay between pulse duration and repetition rate. We see a decrease in length of the absorption zone for increasing pulse durations. The temporal delay between consecutive pulses changes the length of the modification and the strength. Additional experiments foresee the extension to Bessel-like beams and multiple focus spots.
\end{abstract}

DOI: $10.2961 /$ jlmn.2018.03.0022

Keywords: ultrafast optics, laser materials processing, imaging ultrafast phenomena, glass and other amorphous materials

\section{Introduction}

Nowadays, ultrashort laser pulses are suitable candidates for a broad range of applications in various technological fields such as consumer electronics, photonics or medicine [1-3]. Due to the short pulse duration high intensities within the focal region facilitate to locally modify diverse materials ranging from metals, semiconductors to wide-bandgap materials such as glass [4]. However, the corresponding material interaction depends crucially on the different process parameters, e.g., pulse duration, pulse repetition rate, pulse numbers or the total deployed energy [5-7]. Sensitive adjustment of these parameters determines for instance the achievable feed rate or damage of the material. The broad range of accessible applications is complemented by the possibility to use alternative beam shaping concepts instead of the standard Gaussian beam shape. Recent studies investigate the combination of ultrashort laser pulses with Bessel-like beams, aberration corrected profiles for inclined laser light or elongated focus profiles [8-10].

To exploit the full potential for each of the specific applications and derive tailored process strategies, a deep insight into the physics of the fundamental interaction processes and material modifications is of crucial importance. An established technique to investigate linear and nonlinear processes with sub-picosecond temporal resolution is pump-probe microscopy. This method was used in several research areas over the last three decades, which led to constant improvement and a broad field of applications, ranging from biomedical tissue investigation to material characterization [11]. It thus enables to capture the interaction of one or multiple laser pulses inside the material at a given time delay [12].
Another benefit is the possibility to integrate commonly known microscopy techniques. Depending on the observation point and technique for the microscopic images different effects associated to, e.g., carrier excitation, thermalization or their permanent modification are accessible [13]. Recent studies show the combination of tomographic methods and pump-probe microscopy, which provides detailed and spatially extended information [14].

Here, we report on our novel pump-probe system extended to polarization microscopy, which allows the detection of transient stress or pressure waves. We use both microscopy setups to investigate the influence of pulse duration, pulse repetition rate and number of pulses onto the extinction profile and overall stress distribution. Critical process windows and process limitations can be deduced from those experiments. In addition, we apply the experiments to Bessel-like beam profiles and multi-spot arrangements to assess the transferability of the process parameters to different beam shapes.

\section{Experimental Setup}

The pump-probe images were recorded with the setup illustrated in Fig. 1. Two ultrafast sources based on the industrial TRUMPF TruMicro Series 2000 platform are used. Both amplifier modules are fed by the same seed module (50 MHz) but pick their seed pulses independently. Thus, the pulses are synchronized and can be delayed electronically by multiples n of 20 ns.

The processing laser is operated at a wavelength of $\lambda=1030 \mathrm{~nm}$ and the pulse duration can be adjusted continuously by an electronically controlled compressor from $300 \mathrm{fs}$ to $20 \mathrm{ps}$. A subsequent spatial light modulator 


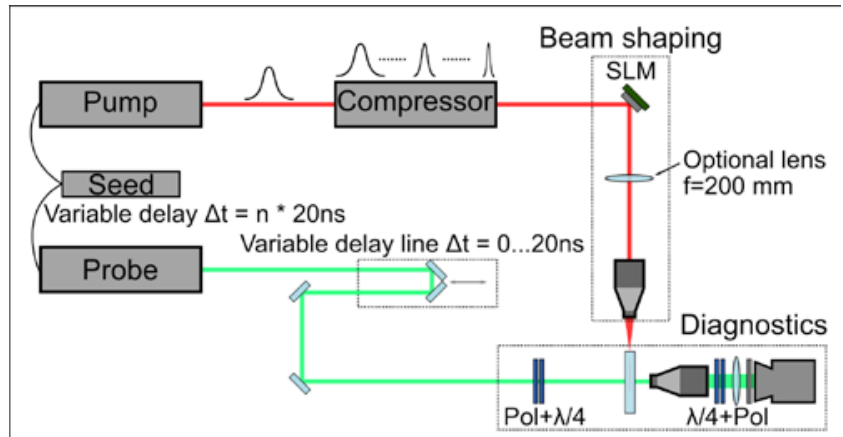

Fig. 1 Sketch of the experimental setup used for in situ diagnostics. Depending on the desired beam profile, an optional lens in the beam shaping segment can be inserted to create a $4 \mathrm{f}$ - setup.

(SLM) with corresponding imaging setup offers the possibility to change the beam shape. Finally, the pump pulse is focused by a microscope objective (NA 0.4, focal length 10 $\mathrm{mm})$ into non-strengthened Corning ${ }^{\circledR}$ Gorilla ${ }^{\circledR}$ Glass.

The second laser pulse (probe) with a pulse duration of approximately $200 \mathrm{fs}$ is frequency doubled $(\lambda=515 \mathrm{~nm})$ and is used to illuminate the interaction area transversely to the processing beam path. The probe laser pulse can be temporally delayed with respect to the pump laser pulse by combining the electronic delay of the seed module with a variable, motor-driven delay line. Thus, it is possible to cover observation delays up to several milliseconds with sub-picosecond resolution. The probe beam is either applied in a transmission light microscope or alternatively in a polarization microscope setup and imaged onto a CMOS camera (IDS UI, shutter speed $18 \mu \mathrm{s}$ ). A preceding bandpass filter (spectral width $\pm 1 \mathrm{~nm}$ ) is used to reduce scattered light.

The images depicted in the inset of Fig. 2(b) and Fig. 3(a)+(b) show the optical depth $\tau=\ln \left(\mathrm{I}_{0} / \mathrm{I}_{\mathrm{S}}\right)$ calculated from shadowgraph images $\left(\mathrm{I}_{\mathrm{S}}\right)$ and their corresponding background images $\left(\mathrm{I}_{0}\right)$. They further express the attenuation of light passing through the material depending on the local extinction coefficient $\kappa$ known from Lambert-Beer's law [15]. The images exhibit reduced probe intensity if probe radiation is absorbed or scattered at excited electrons, defects or transient states. In addition, compression or expansion of the material, i.e. pressure waves or molten mate- rial, can lead to local refractive index changes that may enhance or reduce the corresponding zones in the images [15].

Thus, the false color images indicate regions with high extinction $\kappa$ in bright colors. Dark regions correspond to increased light intensity than in the respective background image, e.g., due to plasma luminescence [16] or refractive index changes leading to lens-like focusing and local concentration of light.

Moreover, the probe beam path was extended with polarization optics to also measure light whose polarization state has changed and thereby analyze transient stress with the described temporal and spatial resolution. In this regard, a circular polariscope, consisting of a polarizer and quarter wave plate before and quarter wave plate and analyzer after the sample serves to measure the optical retardance of the sample within one shot [17]. For calibration of the optical retardance homemade waveplates based on self-assembled nanogratings [18, 19] with known optical retardance (measured with a commercial strain analyzer Ilis StrainMatic M4/60.13) were used. In addition, the pump probe polarization images were background adjusted by subtracting the background noise.

We use the birefringence of a pressure wave caused by a single laser pulse $[15,20,21]$ to verify the functionality of our pump-probe polariscope. A first experiment with the polarization contrast microscope is depicted in Fig. 2. The series shows the birefringence generated by a single laser pulse with 5 ps pulse duration and $13 \mu \mathrm{J}$ pulse energy for different time delays. Figures 2(a)-(d) show the propagation of a pressure wave from 500 ps to 10 ns. Due to the nonlinear induced absorption of the laser pulse and subsequent interaction processes, a free electron plasma is generated, see references $[5,22]$ for detailed description. The plasma leads to a locally confined heating of the lattice. This sudden temperature change causes an increased pressure in the heated area [23]. The start of the corresponding relaxation pressure wave is depicted for one projection in Fig. 2(a). The direction of propagation is radially symmetrical and the amplitude of the pressure wave decreases with increasing distance, see Fig. 2(b)-(d). The inset in Fig. 2(b) shows the observed signal without the circular polariscope, the corresponding scale bar of the optical depth is shown in Fig. 3(a)+(b). Whereas the shape of the absorption profile

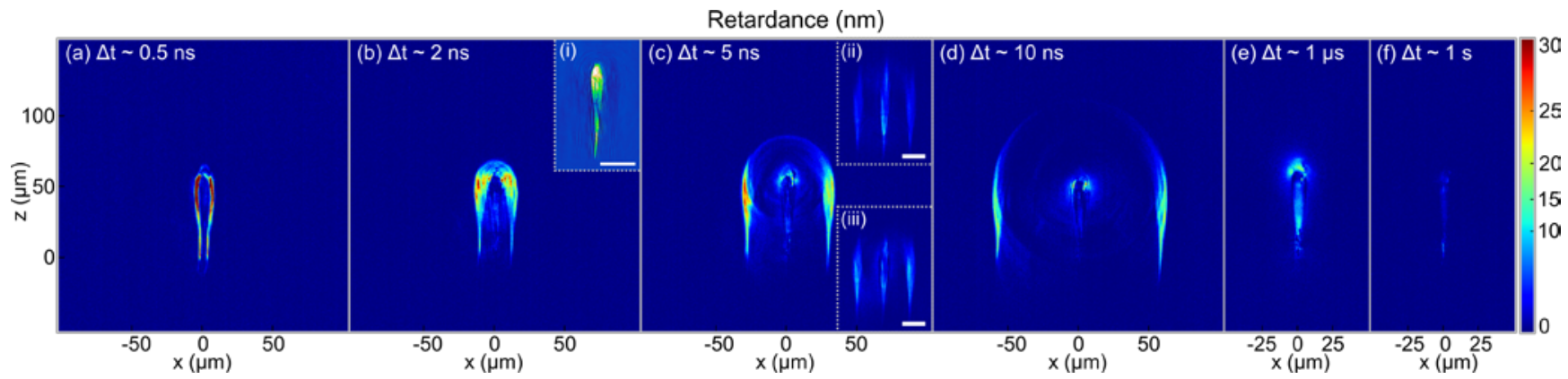

Fig. 2 (a) - (d) Evolution of a pressure wave erupting from the interaction of the material with a single laser pulse of 5 ps pulse duration at a time delay of $0.5 \mathrm{~ns}, 2 \mathrm{~ns}, 5 \mathrm{~ns}$ and $10 \mathrm{~ns}$. The birefringence-profiles in (e) and (f) are recorded after $1 \mu \mathrm{s}$ and $1 \mathrm{~s}$, respectively. The corresponding shadowgraph image for $\Delta \mathrm{t} \sim 2$ ns showing the optical depth is depicted in inset (i), the corresponding scale color-scheme is shown in Fig. 3. The insets (ii) and (iii) show the optical retardance for different pulse durations of $300 \mathrm{fs}$ and 1 ps, respectively, whereby the scale is reduced about a factor of 2 . The scale in (i)-(iii) is $25 \mu \mathrm{m}$. 


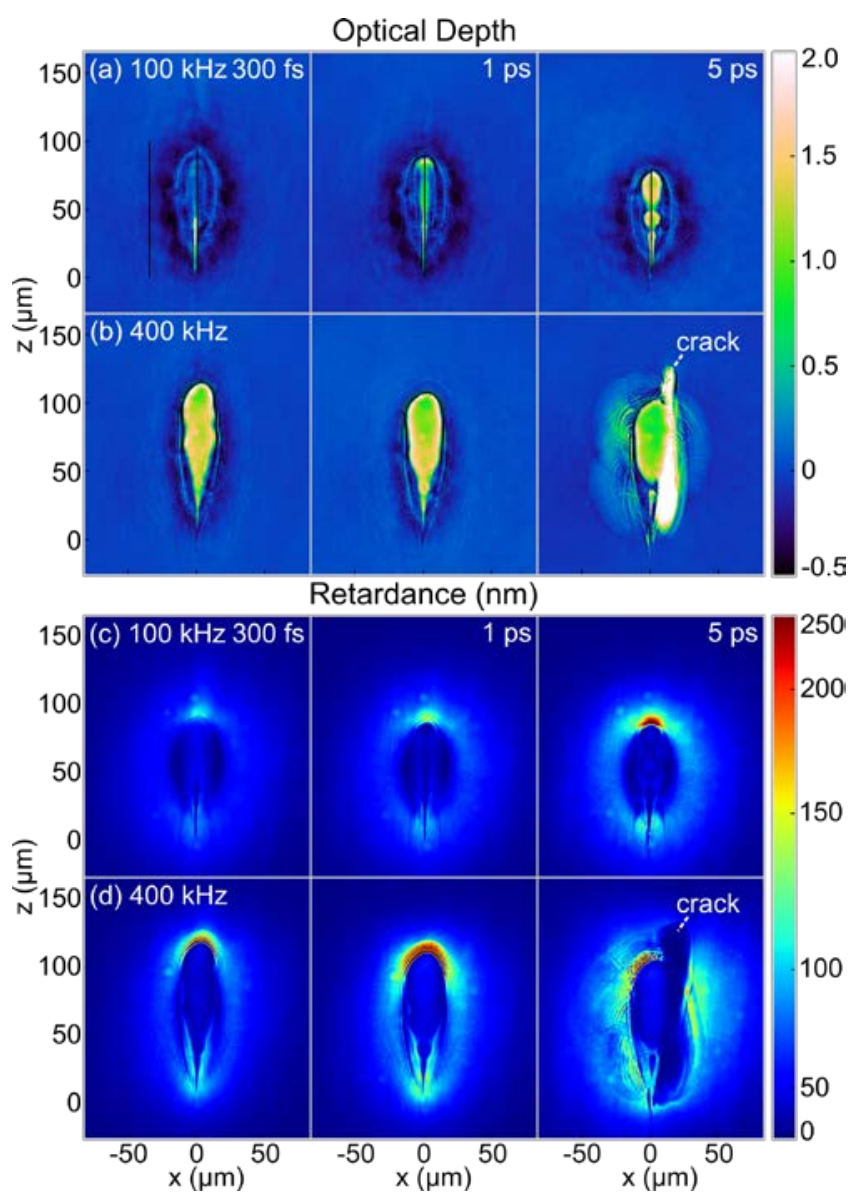

Fig. 3 Shadowgraph images (a)+(b) and polarization microscopy images $(\mathrm{c})+(\mathrm{d})$ captured 2 ns after the last of 50 laser pulses. The respective upper row corresponds to $100 \mathrm{kHz}$, the lower row to $400 \mathrm{kHz}$ pulse repetition rate. The columns from left to right denote the recorded modification using a pulse duration of 300 fs, 1 ps and 5 ps.

is clearly defined, the transient refractive index changes caused by the pressure wave are hardly visible. Figure 2(e) shows the optical retardance on a microsecond timescale. Here, thermally induced stress distributions are still visible. This becomes clear by comparison with the permanent retardance distribution visible in Fig. 2(f), where only small signal is left.

\section{Results and Discussion}

Pump-probe shadow imaging and pump-probe polarization contrast microscopy were used to evaluate the absorption profile and stress distribution of multiple laser pulses inside the glass volume, see Fig. 3 . The images show the corresponding profiles 2 ns after the last of 50 laser pulses. Each measurement was set at a pristine position. The calculated optical depth from measurements with $100 \mathrm{kHz}$ pulse repetition rate exhibits several differences for different pulse durations, cf. Fig. 3(a). The total length of the absorption zone along the $\mathrm{z}$-direction decreases with increasing pulse duration from $\sim 90 \mu \mathrm{m}$ (300 fs) over $\sim 89 \mu \mathrm{m}$ (1 ps) to $\sim 78 \mu \mathrm{m}$ ( $5 \mathrm{ps}$ ) for the measurements after 50 pulses. The origin of this different length can be traced back to the interaction of the first laser pulses with the material. The peak intensity increases for shorter pulse durations and constant pulse energy of $13 \mu \mathrm{J}$.

Nonlinear absorption with subsequent free electron generation first sets in at the focal position, if the pulse intensity exceeds a material specific threshold. Consequently, the higher the initial peak intensity, the farther away from the focus this threshold can be exceeded. Absorption sets in at the geometrical focus and the growth direction is pointed towards the incoming laser pulse [15]. Thus, the modified area is longer for shorter pulse durations.

We therefore assume the initial (nonlinear) absorption of the laser pulse to define the maximum achievable length of the interaction zone and ongoing energy supply of longer lasting pulses to enable further absorption, i.e. avalanche ionization [22], and thus enhance the contrast (higher optical depth value) in the corresponding region. The result is an increased amount of deposited energy for longer pulse durations [6] on the absorption length, pre-defined by the peak intensity. This is supported by the insets in Fig. 2(c) for pulse duration of $300 \mathrm{fs}$ and $1 \mathrm{ps}$ (scale reduced by a factor of 2). The length of the absorption zone is increased, but the strength of the pressure wave is clearly reduced compared to the 5 ps profile. The unequal modification length for different pulse durations is also visible in measurements with multiple pulses, see Fig. 3(a). Here, the accumulation between the laser pulses influences the interaction threshold and the overall length is increased compared to single pulses. In addition, the increase in deposited energy for longer pulse durations [6] results in higher glass temperatures and hence increased heat accumulation leading to a more spherical melt profile via diffusion.

The image series in Fig. 3(b) shows the optical depth for a pulse repetition rate of $400 \mathrm{kHz}$ and otherwise the same process parameters. Here, we observe an increased overall modification length, i.e. from $\sim 90 \mu \mathrm{m}$ at $100 \mathrm{kHz}$ to $\sim 105 \mu \mathrm{m}$ at $400 \mathrm{kHz}$ along the z-direction for pulses with a duration of $300 \mathrm{fs}$. In addition, the size of the area with significant optical depth $(\tau>1)$ increased as well. This is due to the stronger heat accumulation at higher repetition rates while defects such as electronic self-trapping or thermally hindered electronic relaxation promotes absorption of probe light [5, 24].

When comparing different pulse durations, the optical depth increases at $100 \mathrm{kHz}$ with increasing pulse durations. In contrast, at $400 \mathrm{kHz}$ the optical depth within the central modification is visibly higher even at $300 \mathrm{fs}$ and seems to rather decrease with increasing pulse durations, cf. Fig. 3(a)+(b). A contribution to this may also be due to stronger heating at larger pulse duration, which will cause additional thermal radiation. This signal is not filtered completely by the bandpass filter, as measurements with absent probe illumination confirm. The additional (thermally induced) signal adds to the recorded intensity and appears as less extinction, e.g. visible at 5 ps.

The increased deposited energy for the longer pulse duration results in compressive pressure of the surrounding material and crack formation as the longitudinally extended bright line in Fig. 3(b) illustrates. The corresponding polarization contrast images shown in Fig. 3(c)+(d) underline this assumption. For all pulse durations a homogeneous stress distribution around the modified area appears which weakens with increasing distance. At the top and close to 


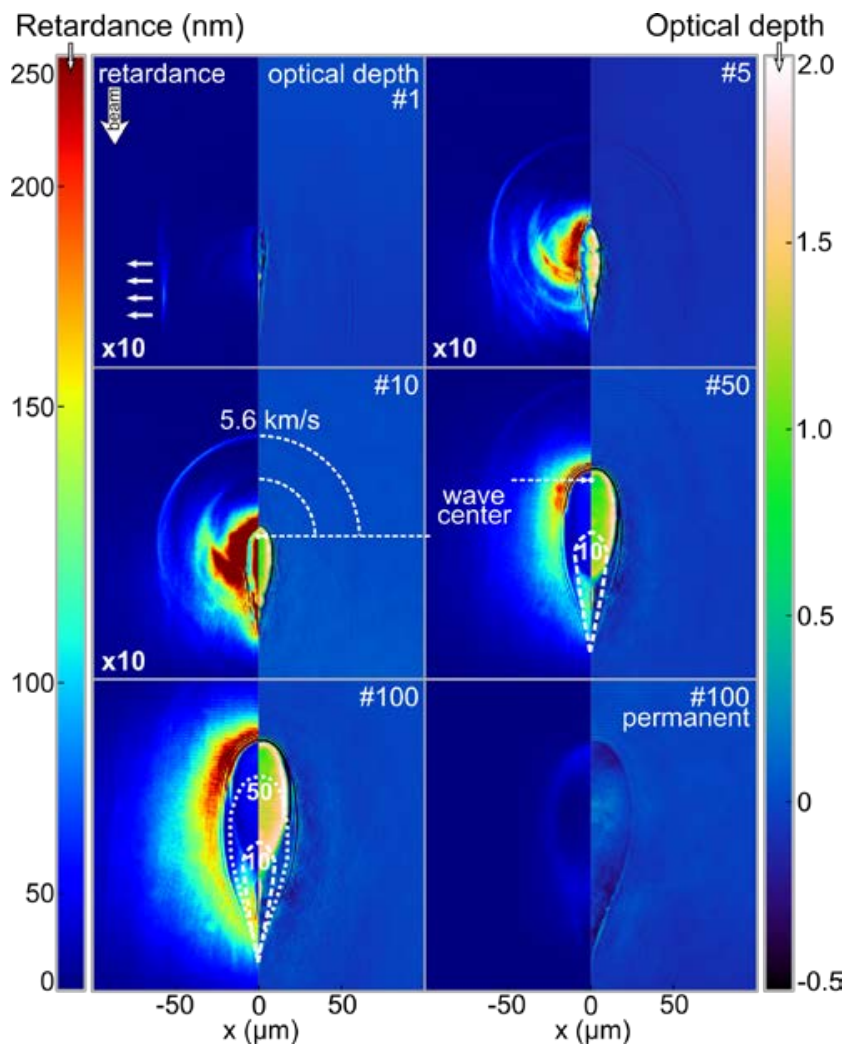

Fig. 4 Images of a two-pulse-burst measurement series (60 ns interval) with 1 ps pulse duration, 10 ns after the last pulse, at a repetition rate of $400 \mathrm{kHz}$. The right side of the sub-image shows the shadowgraph image and the number of pulse trains. The left side shows the measured retardance. The polarization measurements of (\#1)-(\#10) pulse trains are enhanced by a factor of 10 .

the geometrical focus enhanced stress birefringence is measured. Within the central region the material is assumed to be molten releasing any stress that could cause a change of polarization. The strongest retardance for each measurement appears at the top of the modification. Here, significant absorption leading to strong temperature gradients that provoke compressive stress. This effect is pronounced for higher repetition rate due to stronger heat accumulation and hence stronger light absorption [24], cf. Fig. 3(c)+(d). Related experiments conducted at slightly different pulse durations and notable decreased pulse energies by Mermillod-Blondin et al. have shown that the subsequent heating of accumulated pulses leads to an extended on-axis densification, preferably towards the beam incidence [25]. In our experiments, at a pulse duration of 5 ps and a repetition rate of $400 \mathrm{kHz}$, the transient tensile stress becomes too high for the material to withstand. Hence, mechanical relaxation leads to crack development and a drop in retardance signal, as shown in Fig. 3(d).

Profound knowledge of the formation, the avoidance, the enhancement or the healing of cracks is relevant to design cutting or welding applications. Associated with the gradual build-up of stress are the heating and cooling dynamics. Thus, we analyze the absorption and stress distribution for an increasing number of pulses per spot. To enhance the effects of heat accumulation, we use a burst configuration consisting of two pulses with a delay of $60 \mathrm{~ns}$ [26]. The burst energy of $13 \mu \mathrm{J}$ is evenly distributed between the

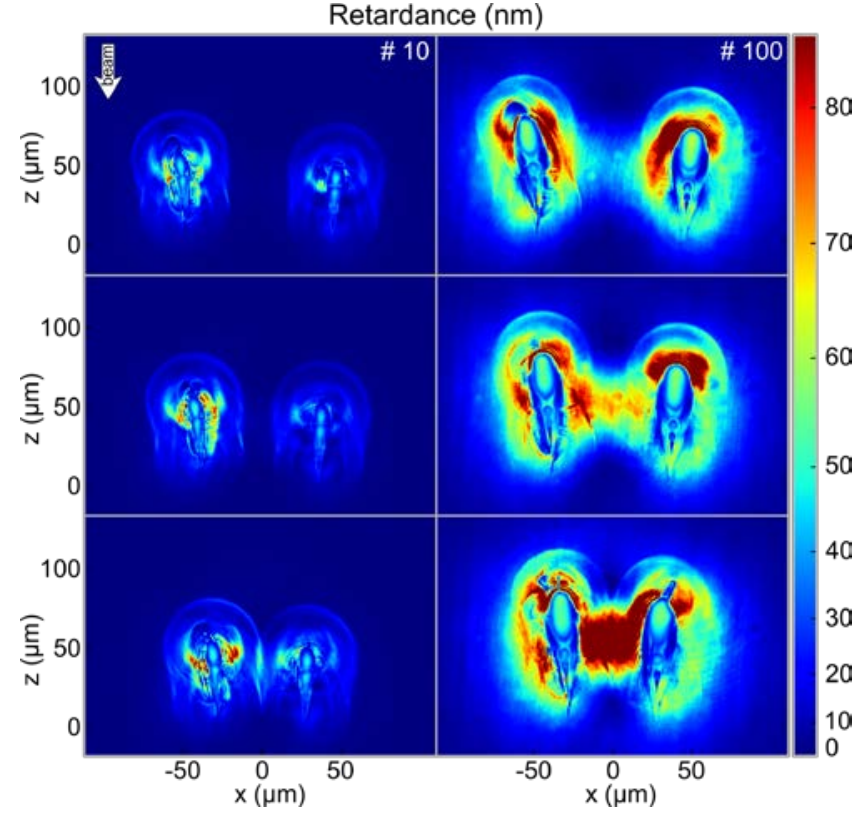

Fig. 5 Arrangement of two Gaussian shaped spots with 5 ps pulse duration at a time delay of $5 \mathrm{~ns}$. The centers of the spots are $\sim 37 \mu \mathrm{m}$ (top row), $\sim 56 \mu \mathrm{m}$ (middle row) and $\sim 76 \mu \mathrm{m}$ (bottom row) apart. The left column shows the birefringence induced by 10 pulses, whereas the measurement for 100 pulses is depicted on the righthand side.

pulses. To avoid damage and crack formation at the high burst repetition rate of $400 \mathrm{kHz}$ the pulse duration is set to $1 \mathrm{ps}$, as shown in the previous section. The image series in Fig. 4 shows the measured retardance (left) and optical depth (right) at a time delay of $10 \mathrm{~ns}$ after the last pulse. Please note the 10 times enhanced scale for the retardance in Fig. 4(\#1)-(\#10). For single pulse illumination, the absorption region is followed by a compressional pressure wave that propagates at a velocity of about $5.6 \mathrm{~km} / \mathrm{s}$. This wave accounts for a longitudinal acoustic wave well known from literature $[15,20]$. A second pressure wave with reduced velocity appears in the polarization measurements for 5 pulse trains and 10 pulse trains, respectively. The reduced velocity can be ascribed to a transversal acoustic wave, typically observed in a longitudinal observation scheme [27].

Analyzing the pressure wave(s), we can derive the areas in which the absorbed energy leads to strong temperature gradients. We see a significant pressure wave propagating along the $x$-axis perpendicular to the incoming beam for a single pulse train, see Fig. 4(\#1). However, the adapted scale in Fig. 2(c) reveals an additional radially spreading propagation direction. Interestingly, the virtual center of the pressure waves shifts towards the incident beam direction for increasing number of pulses, emphasized in Fig. 4(\#10)+(\#50). This is consistent with the previous statement, that significant light is absorbed at the region of beam incidence of the accumulated area. The overall growth of the modified volume for 100 pulse trains is depicted in Fig. 4(\#100) whereby the dotted lines indicate the preceding measured volumes. The upper half shows the previously described features of the melted material. To sustain the liquid phase of the molten area a constant energy input is necessary to compensate the dissipation losses 


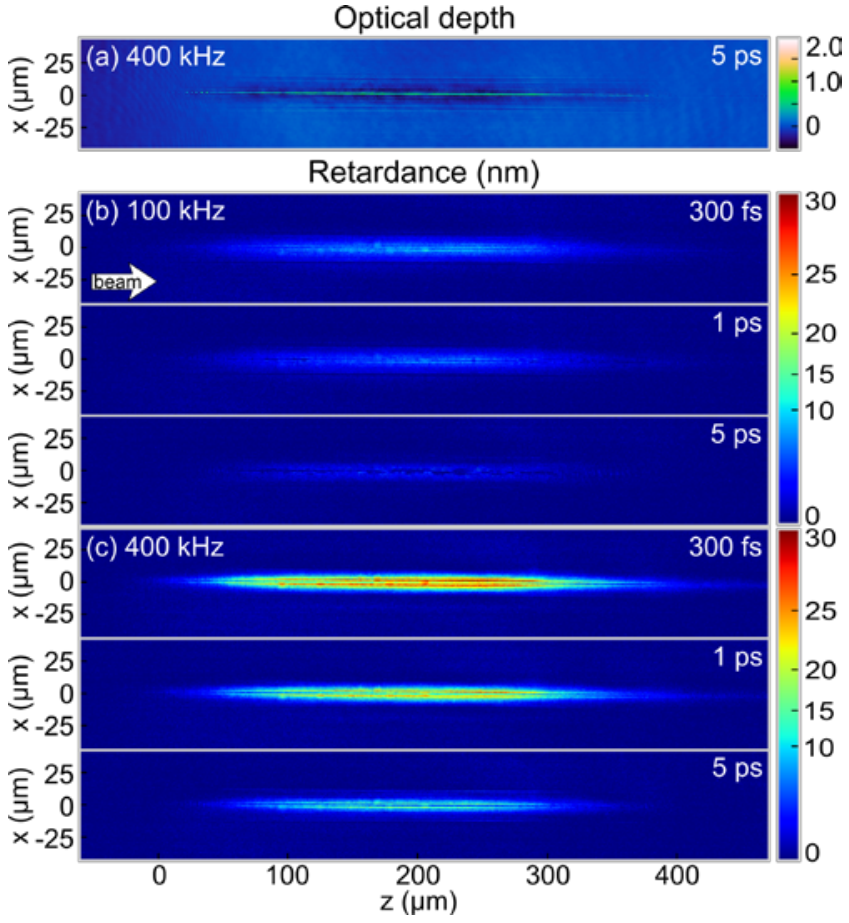

Fig. 6 Polarization microscopy images of Bessel-like beam excitation captured 2 ns after the last of 50 laser pulses for a pulse repetition rate of $100 \mathrm{kHz}$ (a) and $400 \mathrm{kHz}$ (b) with a pulse duration of $300 \mathrm{fs}, 1 \mathrm{ps}$ and 5 ps (top to bottom). Figure (c) shows exemplarily the optical depth for 5 ps pulse duration at $400 \mathrm{kHz}$.

[28]. This condition is met at the top of the modification, visible by the extinction measurement and the increased stress birefringence. The extinction close to the geometrical focus is decimated considerably. Thus, the material starts to resolidify and accompanied stress birefringence inside the volume is observed. Small retardance remains permanently on the outline of the former molten zone, visible in the ex situ measurement in Fig. 4.

The established pump probe setup can also be used to study more advanced beam profiles. For industrial purposes multiplexing is a common approach. One possible application is to influence the crack length generation in crystals [29]. Another possibility foresees to use the available average power of modern ultrashort laser sources, e.g. for diverse applications such as welding. In this regard, two Gaussian beams were focused next to each other in the bulk. Figure 5 shows the polarization contrast images 5 ns after the last laser pulse was present (10 laser pulses in the left and 100 pulses in the right column). The laser parameters are set to a pulse duration of $1 \mathrm{ps}$ at a repetition rate of $400 \mathrm{kHz}$. The pulse energy of $13 \mu \mathrm{J}$ is evenly distributed between the two spots.

The polarization contrast images indicate the two main contributions as observed before: pressure wave emission and compressive stress in the surrounding of the modification due to thermal expansion of the heated material. Here the induced modifications appear slightly tilted with respect to the beam propagation direction better visible at 100 pulses. This is due to a slight defocus aberration as confirmed by beam propagation simulations, as shown in
Appendix A. However, the tilted modification confirms that the strong temperature gradients at the top towards the beam propagation direction trigger strong transient stress in the surrounding and may result in crack formation (e.g. for 10 and 100 pulses in the lowest micrograph).

When the distance of the beam profiles decreases (from upper to lower row in Fig. 4) the stress birefringence of the individual modifications starts to overlap resulting in a strong retardance signal. In case of lower pulse numbers, the overlapping pressure waves result in a pronounced but confined region of transient stress. For larger pulse numbers this region of elevated stress birefringence widens. As observed before (see Fig. 3) this transient stress may result in material failure and can be set by the amount of heat accumulation. This effect emphasizes the potential of tailored beam profiles, e.g. for cutting applications with desired crack propagation direction.

The shown Gaussian-shaped beam profiles are suitable for localized processes, e.g., welding applications. However, elongated beam profiles are preferred in separation processes because they allow single pass modifications for glass thicknesses up to several millimeters. Bessel-like beams fulfill such a high aspect ratio, see the extinction measurement in Fig. 6(a), and are commonly used for cutting applications [9, 30].

The Bessel-like beams were generated by the SLM-setup (see Fig. 1) and subsequently imaged into the glass. The same laser settings as in the preliminary experiments depicted in Fig. 3 are used to allow a comparison between the different beam shapes.

Figure 6(b)+(c) show the polariscope images measured at a temporal delay of 2 ns. Again, the same influence of pulse repetition rate and pulse duration discussed for the Gaussian shaped pulses becomes evident. The stress birefringence increases with higher repetition rates due to stronger heat accumulation. In addition, the length of the modified area decreases with increasing pulse duration. An increase in pulse duration also leads to more birefringence with Bessel-like beams, as single pulse experiments confirm, as shown in Appendix B. Interestingly, the overall measured birefringence decreases for increasing pulse duration at higher pulse numbers, cf. Fig. 6(b)+(c). This might be due to incipient material damage which influences the absorption of subsequent pulses, visible e.g. for 5 ps pulse duration in Fig. 6(b). Here the inside of the absorption zone shows uneven and stress-free areas that contrast with the expected linear extinction profile of Bessel-like beams, cf. Fig. 6(a). Additionally, ex situ measurements show permanent retardance in these areas.

In contrast to the Gaussian-shaped beam profiles, the stress distribution is homogeneous. The reason is explained in the generation of the Bessel-like beams. These are composed of many partial beams which are parallel and pass the material interface under a specific offset angle, see reference [9] for a detailed description. Thus, there is no clear direction of incidence as with Gaussian beam profiles and the aggregated stress growth is homogeneously distributed. In addition, we see no shift towards the beam entrance side, which makes Bessel-beams suitable profiles for cutting applications at high repetition rates. 


\section{Conclusion}

We successfully observed the transient interaction of multiple laser pulses inside glass with the help of in situ pumpprobe microscopy. Additional information was accessible by the newly implemented polarization microscope into our measurement setup. Both diagnostic methods were used to elucidate the different behavior of effects like absorption and transient stress for various laser parameters. The pulse repetition rate was identified to influence the growth and the extend of significant stress under accumulation. Concomitant, the measurements have been extended to multiplexed beam shapes and Bessel-like beams which are promising concepts for welding and cutting applications. Pump-probe polarization microscopy is a powerful research tool to gain access to transient birefringence effects and develop tailored beam profiles with intended stress distributions.

\section{Acknowledgments}

This work is supported by the Federal Ministry of Education and Research (BMBF) (13N13927, 13N13930). We thank TRUMPF Laser GmbH Schramberg for their kind support.

\section{Appendix A}

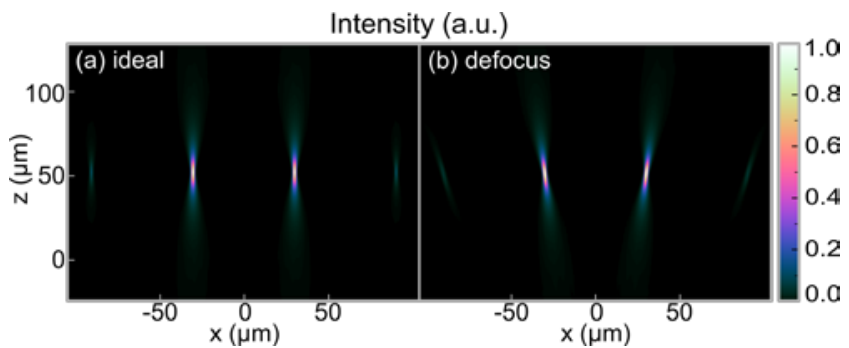

App. A Simulated intensity distribution of two Gaussian shaped laser pulses (a) in the focal plane and (b) with defocus aberration.

\section{Appendix B}

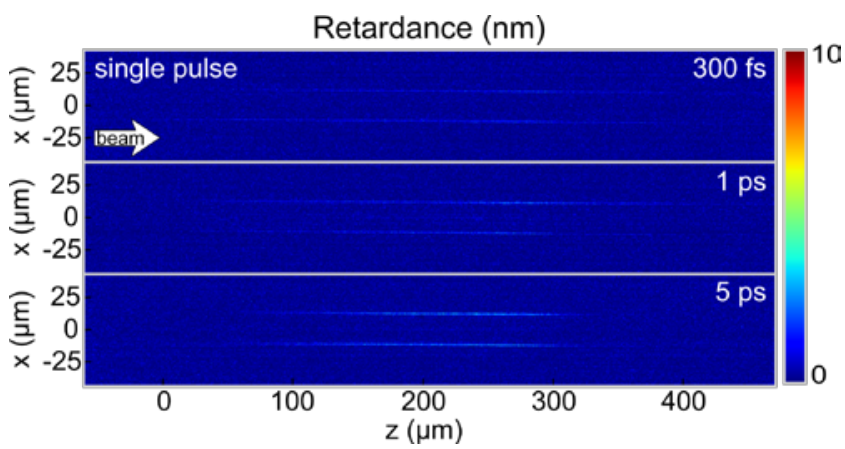

App. B Single pulse polarization microscopy images of Bessel-like beam excitation captured with a temporal delay of $2 \mathrm{~ns}$. The pulse energy was set to $13 \mu \mathrm{J}$ for each pulse duration.

\section{References}

[1] M. Malinauskas, A. Žukauskas, S. Hasegawa, Y. Hayasaki, V. Mizeikis, R. Buividas, and S. Juadkazis: Light Sci. Appl., 5, (2016) e16133.

[2] L. Jiang, A. D. Wang, B. Li, T. H. Cui, and Y. F. Lu: Light Sci. Appl., 7, (2018) 17134.

[3] H. Lubatschowski, A. Heisterkamp, F. Will, A. I. Singh, J. Serbin, A. Ostendorf, O. Kermani, R. Heermann, H. Welling, and W. Ertmer: Riken Rev., 50, (2003) 113.

[4] K. Itoh, W. Watanabe, S. Nolte, and C. B. Schaffer: MRS bull., 31, (2006) 620.

[5] R. R. Gattass, and E. Mazur: Nat. photonics, 2, (2008) 219.

[6] J. Peng, D. Grojo, D. M. Rayner, and P. B. Corkum: Appl. Phys. Lett., 102, (2013) 161105.

[7] D. Tan, K. N. Sharafudeen, Y. Yue, and J. Qiu: Prog. Mater. Sci., 76, (2016) 154.

[8] K. Mishchik, R. Beuton, O. D. Caulier, S. Skupin, B. Chimier, G. Duchateau, B. Chassagne, R. Kling, C. Hönninger, E. Mottay, and J. Lopez: Opt. Expr., 25, (2017) 33271.

[9] M. Jenne, D. Flamm, T. Ouaj, J. Hellstern, J. Kleiner, D. Grossmann, M. Koschig, M. Kaiser, M. Kumkar, and S. Nolte: Opt. Lett., 43, (2018) 3164.

[10] L. Rapp, R. Meyer, L. Furfaro, C. Billet, R. Giust, and F. Courvoisier: Opt. Expr., 25, (2017) 9312.

[11] M. C. Fischer, J. W. Wilson, F. E. Robles, and W. S. Warren: Rev. Sci. Instrum., 87, (2016) 031101.

[12]D. Zimdars, A. Tokmakoff, S. Chen, S. R. Greenfield, M. D. Fayer, T. I. Smith, and H. A. Schwettman: Phys. Rev. Lett., 70, (1993) 2718.

[13] Y. Hayasaki, K. Iwata, S. Hasegawa, A. Takita, and S. Juodkazis: Opt. Mat. Expr., 1, (2011) 1399.

[14]K. Bergner, D. Flamm, M. Jenne, M. Kumkar, A. Tünnermann, and S. Nolte: Opt. Expr., 26, (2018) 2873.

[15]D. Grossmann, M. Reininghaus, C. Kalupka, M. Kumkar, and R. Poprawe: Opt. Expr., 24, (2016) 23221.

[16] D. Grossmann, M. Reininghaus, C. Kalupka, M. Jenne, and M. Kumkar: Opt. Expr., 25, (2017) 28478.

[17] M. Shribak, and R. Oldenbourg: Appl. Optics, 42, (2003) 3009.

[18] R. Taylor, C. Hnatovsky, and E. Simova, Laser Photonics Rev., 2, (2008) 26.

[19]F. Zimmermann, A. Plech, S. Richter, A. Tünnermann, and S. Nolte, Laser Photonics Rev., 10, (2016) 327.

[20] K. Bergner, B. Seyfarth, K. A. Lammers, T. Ullsperger, S. Döring, M. Heinrich, M. Kumkar, D. Flamm, A. Tünnermann, and S. Nolte: Appl. Optics, 57, (2018) 4618.

[21] T. Tochio, M. Sakakura, Y. Shimotsume, M. Nishi, K. Hirao, and K. Miura: Jpn. J. Appl. Phys., 51, (2012) 126602.

[22] B. Rethfeld: Phys. Rev. B, 73, (2006) 035101.

[23] M. Sakakura, and M. Terazima: Phys. Rev. B, 71, (2005) 024113.

[24] I. Miyamoto, Y. Okamoto, R. Tanabe, Y. Ito, K. Cvecek and M. Schmidt: Opt. Expr., 24, (2016) 24718. 
[25] A. Mermillod-Blondin, I. M. Burakov, Y.P. Meshcheryakov, N. M. Bulgakova, E. Audouard, A. Rosenfeld, A. Husakou, I.V. Hertel, and R. Stoian: Phys. Rev. B, 77, (2008) 104205.

[26] S. Richter, F. Zimmermann, R. Eberhardt, A. Tünnermann, and S. Nolte: Appl. Phys. A, 121, (2015) 1.

[27]F. Hendricks, V. V. Matylitsky, M. Domke, and H. P. Huber: Proc. SPIE, (2016) 97401A.

[28] S. Nolte, M. Will, J. Burghoff, and A. Tünnermann: J. Mod. Optic, 51, (2004) 2533.

[29] M. Sakakura, Y. Ishiguro, N. Fukuda, Y. Shimotsuma, and K. Miura: J. Laser Micro Nanoen., 9, (2014) 15.

[30] M. K. Bhuyan, F. Courvoisier, P. A. Lacourt, M. Jacquot, R. Salut, L. Furfaro, and J. M. Dudley: App. Phys. Lett., 97, (2010) 081102.

(Received: July 16, 2018, Accepted: November 11, 2018) 